\title{
Persistent Atrial Fibrillation Ablation in a Case of Persistent Left Superior Vena Cava with Absence of the Right Superior Vena Cava
}

\author{
Tolga Aksu, Tumer Erdem Guler, Serdar Bozyel, Kivanc Yalin \\ Usak University, Usak, Turkey \\ ORCID: \\ Tolga Aksu: https://orcid.org/0000-0001-8061-9660 \\ Tümer Erdem Güler: https://orcid.org/0000-0003-1349-2123 \\ Serdar Bozyel: https://orcid.org/0000-0002-6582-3660 \\ KIvanç Yalın: https://orcid.org/0000-0001-5602-0711
}

Department of Cardiology, Kocaeli Derince Training and Research Hospital, University of Health Sciences, Kocaeli, 'Department of Cardiology, Faculty of Medicine,

\section{Abstract}

The great majority of atrial fibrillation cases originate from pulmonary vein (PV) triggers; however, non-PV triggers may be the drivers of the arrhythmia, especially in the patients with a persistent left superior vena cava (PLSVC). The presence of a PLSVC may be suggested with a dilated coronary sinus on transesophageal echocardiography during the procedure and may be confirmed with an atrial angiogram, if not known beforehand. Detection and elimination of true trigger site are the most important step for clinical success in such cases.

Keywords: Ablation, atrial fibrillation, persistent left superior vena cava, transesophageal echocardiography

\section{INTRODUCTION}

On the basis of modern knowledge, pulmonary vein (PV) and non-PV triggers consist of main target in atrial fibrillation (AF) ablation. The role of persistent left superior vena cava (PLSVC) in etiopathogenesis of AF is still sparse. This report presents the reasonable approach to ablation of persistent $\mathrm{AF}$ in a case of PLSVC with the absence of right superior vena cava (RSVC).

\section{Case Report}

A 58-year-old female with a history of drug refractory symptomatic AF for 6 months was referred to our institution for ablation. Transthoracic echocardiography demonstrated normal cardiac structure, and physical examination was unremarkable. Preablation transesophageal echocardiography (TEE) was deferred because of otherwise relatively low-risk features at the time of ablation (paroxysmal AF substrate, presentation

Submission: 20-Dec-18 Revision: 18-Jan-19 Accepted: 20-Jan-19

\begin{tabular}{|l|l|}
\hline \multicolumn{3}{c|}{ Access this article online } \\
\hline Quick Response Code: & Website: \\
& \\
http://www.ijcva.com
\end{tabular}

in sinus rhythm, and the patient being on uninterrupted rivaroxaban).

After placement of a duodecapolar coronary sinus catheter, a J-tipped guidewire could not be advanced to the RSVC during fluoroscopically guided transseptal puncture attempt. To allow direct visualization of the transseptal needle tip within the fossa ovalis, we decided to use TEE guidance which demonstrated the absence of RSVC and dilated coronary sinus. To reveal cardiac venous anatomy, an angiogram of the right atrium was performed and confirmed the existence of PLSVC with absent of RSVC [Figure 1a and b].

Address for correspondence: Dr. Tolga Aksu, Department of Cardiology, Kocaeli Derince Education and Research Hospital, University of Health Sciences, Kocaeli 41500, Turkey. E-mail: aksutolga@gmail.com

This is an open access journal, and articles are distributed under the terms of the Creative Commons Attribution-NonCommercial-ShareAlike 4.0 License, which allows others to remix, tweak, and build upon the work non-commercially, as long as appropriate credit is given and the new creations are licensed under the identical terms.

For reprints contact: reprints@medknow.com

How to cite this article: Aksu T, Guler TE, Bozyel S, Yalin K. Persistent atrial fibrillation ablation in a case of persistent left superior vena cava with absence of the right superior vena cava. Int J Cardiovasc Acad 2019;5:68-70 
Transseptal puncture was performed under the guidance of TEE, and a cryoablation (CA) catheter was inserted into the left superior PV. Before starting CA in the PVs, a duodecapolar catheter was inserted retrograde through the coronary sinus into the PLSVC. Entrance block was observed in PLSVC which confirmed AF was not triggered by PLSVC [Figure 2]. During CA of the left superior PV, AF was first organized and

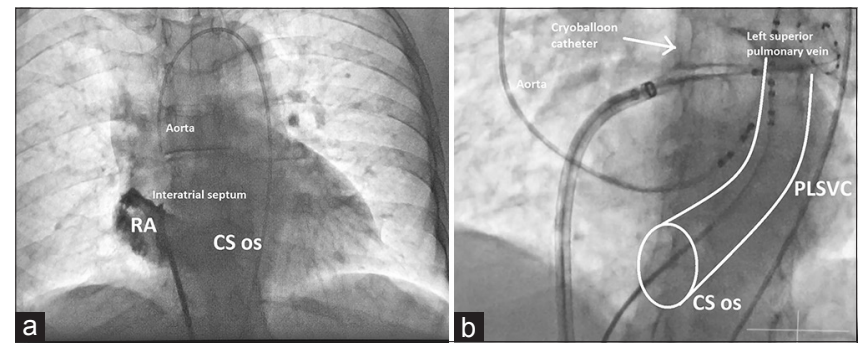

Figure 1: A left anterior oblique fluoroscopic view of the spine. (a) The angiogram of the RA demonstrates absence of a right-sided superior vena cava. (b) A coronary sinus catheter is placed in the PLSVC. The border of CS OS and PLSVC was indicated with white lines. CS OS: Coronary sinus ostium, PLSVC: Persistent left superior vena cava, RA: Right atrium

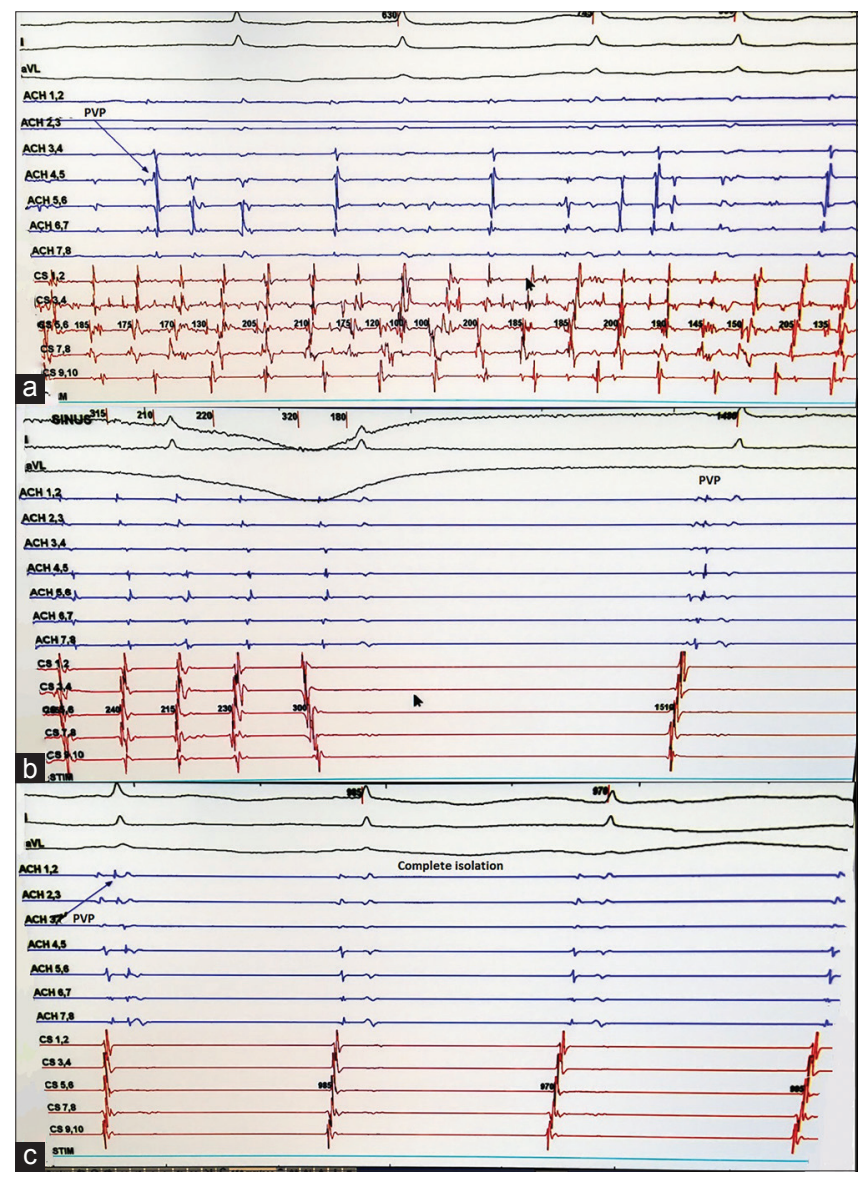

Figure 3: Intracardiac recordings of the left superior pulmonary vein. (a) Atrial fibrillation is seen at the beginning of cryoablation in the left superior pulmonary vein. (b) Atrial fibrillation is organized and terminated while ablation is in progress. Please take care that pulmonary vein potential is still seen in the last beat. (c) Isolation of pulmonary vein is achieved in the second beat then terminated to sinus rhythm [Figure 3a-c]. Other PVs were isolated using a double-freeze method with each application lasting for $180 \mathrm{~s}$. After completion of PV isolation, the pacing was performed through PLSVC with maximal energy. It verified the existence of exit block. Dormant conduction was not observed and burst pacing attempts did not induce AF with isoproterenol infusion $(3.3 \mu \mathrm{g} / \mathrm{min})$. Postprocedural computed tomography angiography confirmed the existence of PLSVC with the absence of RSVC [Figure $4 \mathrm{a}$ and $\mathrm{b}$ ].

\section{Discussion}

The dominant trigger for AF is usually PVs although non-PV foci may explain arrhythmia recurrence in some patients after PV isolation. ${ }^{[1,2]}$ In the presence of PLSVC which may contain remnant muscular and pacemaker tissue carried over from embryonic life, it may contain trigger sites for initiation of AF. ${ }^{[3]}$ Possible electrical connections between PLSVC to the coronary sinus and to the left atrium are considered the main cause of arrhythmogenic properties of PLSVC. Despite these anatomical considerations, we detected an entrance block in PLSVC during AF which confirmed that AF was not triggered by PLSVC in our case. Therefore, we did not perform ablation in PLSVC. If PLSVC was detected the trigger site for AF, focal ablation in trigger site or electrical isolation of PLSVC should be attempted. ${ }^{[3,4]}$

Although transseptal puncture under fluoroscopic guidance may have a reasonable safety profile in experienced hands, serious

1.5P PLSVC

Figure 2: Intracardiac recordings of the PLSVC. There is no atrial electrogram in the left superior vena cava which suggests entrance block. PLSVC: Persistent left superior vena cava
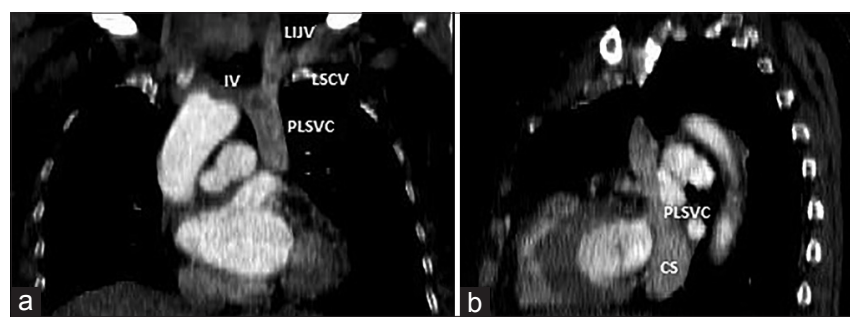

Figure 4: Contrast-enhanced coronal chest computed tomography images. ( $a$ and $b$ ) The images demonstrate the presence of persistent left superior vena cava and absence of a right-sided superior vena cava. CS: Coronary sinus, IV: Innominate vein, LIJV: Left internal jugular vein, LSCV: Left subclavian vein, PLSVC: Persistent left superior vena cava 
complications such as cardiac tamponade $(1.31 \%)$ or aortic perforation can still occur and can lead to death $(0.15 \%) \cdot{ }^{[5,6]}$ To deal with these possible complications, transseptal puncture should be done under TEE or intracardiac echocardiography guidance. The use of echocardiographic guidance for transseptal puncture does not only enable a safe procedure but also enables puncture site selection within the fossa ovalis according to the expected procedure type (e.g., a more anterior puncture for ablation of an accessory pathway at the mitral annulus or more posterior puncture for ablation of AF). Furthermore, the possibility of safely initiation of anticoagulation before transseptal puncture may be another important advantage of echocardiographic guidance. ${ }^{[7]}$

As a conclusion, PLSVC is an anomalous structure may cause procedural challenge and additionally may serve as a substrate for AF. Despite, the dominant trigger for AF is usually non-PVs in PLSVC cases; it should be kept in mind to check electrical activity of anomalous PLSVC before attempting empirical ablation.

\section{Declaration of patient consent}

The authors certify that they have obtained all appropriate patient consent forms. In the form the patient(s) has/have given his/her/their consent for his/her/their images and other clinical information to be reported in the journal. The patients understand that their names and initials will not be published and due efforts will be made to conceal their identity, but anonymity cannot be guaranteed.

\section{Financial support and sponsorship}

Nil.

Conflicts of interest

There are no conflicts of interest.

\section{RefEREnCES}

1. Haïssaguerre M, Jaïs P, Shah DC, Takahashi A, Hocini M, Quiniou G, et al. Spontaneous initiation of atrial fibrillation by ectopic beats originating in the pulmonary veins. N Engl J Med 1998; 339:659-66.

2. Shah $D$, Haissaguerre M, Jais P, Hocini M. Nonpulmonary vein foci: Do they exist? Pacing Clin Electrophysiol 2003;26:1631-5.

3. Gaita F, Scaglione M, Ferraris F. Left persistent superior vena cava as a source of focal atrial fibrillation. Eur Heart J 2010;31:1689.

4. Abrich VA, Munro J, Srivathsan K. Atrial fibrillation ablation with persistent left superior vena cava detected during intracardiac echocardiography. HeartRhythm Case Rep 2017;3:455-8.

5. De Ponti R, Cappato R, Curnis A, Della Bella P, Padeletti L, Raviele A, et al. Trans-septal catheterization in the electrophysiology laboratory: Data from a multicenter survey spanning 12 years. J Am Coll Cardiol 2006;47:1037-42.

6. Kautzner J, Peichl P. You get what you inspect, not what you expect: Can we make the transseptal puncture safer? Europace 2010;12:1353-5.

7. Erden I, Erden EÇ, Golcuk E, Aksu T, Yalin K, Güler TE, et al. Impact of transesophageal echocardiography during transseptal puncture on atrial fibrillation ablation. J Arrhythm 2016;32:170-5. 